\title{
Silver/titania nanocomposite-modified photoelectrodes for photoelectrocatalytic methanol oxidation
}

\begin{abstract}
Silver deposited titania $(\mathrm{Ag} / \mathrm{TiO} 2)$ nanocomposite thin films were fabricated by the simple sonochemical deposition of Ag on preformed aerosol-assisted chemical vapor deposited TiO2 thin films. The photelectrocatalytic performance of a newly fabricated Ag/TiO2-modified photoelectrode was studied for methanol oxidation under simulated solar AM $1.5 \mathrm{G}$ irradiation $(100 \mathrm{~mW} / \mathrm{cm} 2)$. The $\mathrm{Ag} / \mathrm{TiO} 2$-modified photoelectrode showed a photocurrent density of $1 \mathrm{~mA} / \mathrm{cm} 2$, which is four times that of an unmodified $\mathrm{TiO} 2$ photoelectrode. The modification of $\mathrm{Ag}$ on the $\mathrm{TiO} 2$ surface significantly enhanced the photoelectrocatalytic performance by improving the interfacial charge transfer processes, which minimized the charge recombination. Density functional theory (DFT) calculation studies revealed that methanol could be easily adsorbed onto the Ag surfaces of $\mathrm{Ag} / \mathrm{TiO} 2$ via a partial electron transfer from Ag to methanol. The newly fabricated Ag/TiO2-modified photoelectrode could be a promising candidate for photoelectrochemical applications.
\end{abstract}

Keyword: Ag/TiO2; Thin film; Photoelectrochemistry; AACVD; Methanol oxidation 